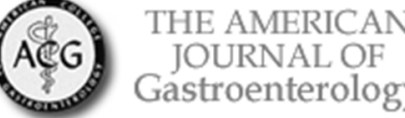

\title{
Bacterial infections change natural history of cirrhosis irrespective of liver disease severity
}

\begin{tabular}{|r|l|}
\hline Journal: & American Journal of Gastroenterology \\
\hline Manuscript ID & AJG-16-1783.R1 \\
\hline Manuscript Type: & Original Contributions \\
\hline Keywords: & decompensation, sepsis, prognostic score, survival \\
\hline Manuscript Section: & Liver \\
\hline \multicolumn{2}{|l}{} \\
\hline
\end{tabular}

SCHOLARONE ${ }^{\mathrm{m}}$

Manuscripts 


\section{Bacterial infections change natural history of cirrhosis irrespective of liver disease} severity

Elena Dionigi1,2, Matteo Garcovich¹, Mauro Borzio², Gioacchino Leandro ${ }^{3}$, Avik Majumdar1, Aikaterini Tsami $^{1}$, Vasiliki Arvaniti ${ }^{1}$, Davide Roccarina ${ }^{1}$, Massimo Pinzani ${ }^{1}$, Andrew K. Burroughs $^{1}$, James O’Beirne ${ }^{1 *}$, Emmanuel A. Tsochatzis ${ }^{1^{*}}$

1. UCL Institute for Liver and Digestive Health and Sheila Sherlock Liver Unit, Royal Free Hospital and UCL, London, UK

2. Unità di Gastroenterologia, Azienda Ospedaliera di Melegnano, 20070 Vizzolo Predabissi, Italy

3. Gastroenterology I Department, IRCCS De Bellis Hospital, Castellana Grotte, Italy

*These two authors contributed equally to this manuscript and are joint senior authors

Correspondence: Emmanuel A. Tsochatzis, Sheila Sherlock Liver Unit and UCL Institute of Liver and Digestive Health, Royal Free Hospital and UCL, Pond Street, NW3 2QG, London, UK, Phone: (0044)2077940500 ext 31142, Fax: (0044)2074726226, email: e.tsochatzis@ucl.ac.uk

Keywords: decompensation; sepsis; prognostic score; survival

Abbreviations: OLT orthotopic liver transplant; MELD Model of End-stage Liver Disease; OR odds ratio; ITU intensive care; INR international normalized ratio; HIV human immunodeficiency virus; UKELD United Kingdom Model for End-stage Liver Disease; HBV hepatitis B virus; HCV hepatitis C virus; SBP Spontaneous bacterial peritonitis; SE spontaneous empyema; UTI urinary tract infection; CFU colony forming unit; MRSA 
methicillin resistant staphylococcus aureus; CA community acquired; HCA Health-care associated; SD standard deviation; NASH non-alcoholic steato-hepatitis; TIPSS transjugular portosystemic shunt; HCC hepatocellular carcinoma; ESBL Extended Spectrum $\beta$-Lactamase; CPS Child-Pugh score; MDRB Multidrug Resistant Bacteria

Electronic word count: 5955

Tables: 5

Figures: 2

Conflicts of interest: None

Financial support: None

Authors contributions: ED data collection, analysis and interpretation, drafting the manuscript, MG, AT, VA, AM, DR data collection, GL data analysis, MB, MP critical revision. AKB conception and design, JOB, EAT conception and design, critical revision. All authors approved the final version. 
What is already known:

- Bacterial infections are common in patients with cirrhosis

- Bacterial infections worsen the prognosis of hospitalized patients with cirrhosis

- The role of infections in changing the natural history of patients with cirrhosis has been hypothesized

What is new here:

- Patients with cirrhosis who become infected have a greater risk of death even if they survive the acute episode of infection, independently of the severity of their underlying liver disease

- A single episode of infection was independently associated with survival over and above the scores used in transplant centers and degree of liver disease severity

- Infection most likely represents a distinct prognostic stage of cirrhosis, which affects survival irrespective of disease severity 


\section{Abstract}

Background/aims: We assessed the prognostic significance of infections in relation to current prognostic scores and explored if infection could be considered per se a distinct clinical stage in the natural history of cirrhosis.

Methods: We included consecutive patients with cirrhosis admitted to a tertiary referral liver unit for at least 48 hours over a 2-year period. Diagnosis of infection was based on positive cultures or strict established criteria. We used competing risk analysis and propensity score matching for data analysis.

Results: 501 patients (63\% male, 48\% alcoholic liver disease, median MELD=17) underwent 781 admissions over the study period. Portal hypertensive bleeding and complicated ascites were the commonest reasons of admission. The incidence of proven bacterial infection was $25.6 \%$ (60\% community acquired and 40\% nosocomial). Survival rates at 3, 6, 12 and 30 months were $83 \%, 77 \%, 71 \%, 62 \%$ in patients without diagnosis of infection, versus $50 \%$, $46 \%, 41 \%$ and $34 \%$ in patients with diagnosis of infection. Overall survival was independently associated with MELD score (HR 1.099), ITU stay (HR 1.967) and bacterial infection (HR 2.226). Bacterial infection was an independent predictor of survival even when patients who died within the first 30 days were excluded from the analysis in Cox regression (HR 2.013) and competing risk Cox models in all patients (HR 1.46) and propensity risk scorematched infected and non-infected patients (HR 1.67).

Conclusions: Infection most likely represents a distinct prognostic stage of cirrhosis, which affects survival irrespective of disease severity, even after recovery from the infective episode. 
It is well established that bacterial infections are common in patients with cirrhosis and carry a poor prognosis (1). More worryingly, the prevalence of infections by multi-resistant bacteria is also increasing (2). Recently, the role of infections in changing the natural history of patients with cirrhosis has been hypothesized, and the occurrence of infection in patients with cirrhosis has been proposed as a separate clinical stage, based on a meta-analysis of 178 studies (3).

Among patients with cirrhosis on the transplant waiting list, prioritization is based on predictive scores based primarily on liver disease severity. The relative shortage of organ donors renders allocation algorithms of organs essential, and the optimal strategy based on scoring systems or waiting time is still under debate.

The Model for End Stage Liver Disease (MELD) score incorporates serum creatinine, serum total bilirubin and INR and is widely used to predict the short-term mortality in patients with cirrhosis (4). Many studies have shown its capability to correctly rank potential liver recipients according to their severity of liver disease and mortality risk, however, potential limitations of this score have been reported $(5,6)$. Despite their widespread application, the prognostic accuracy of all these models is far from being satisfactory and a large number of patients still die while on the transplant waiting list, and sometimes with MELD scores below the threshold of transplantation.

D'Amico recently proposed a sub-classification of cirrhosis based on clinical stages with distinct one-year mortality that ranges from $1-57 \%$ depending on the stage (7). This classification is simple and reproducible, defined by clinical criteria that occur in the natural history of cirrhosis such as presence of varices, ascites and variceal bleeding and have a different prognosis. Infection was proposed as a further stage in this classification (3).

The aim of this study was twofold, firstly to confirm the prognostic importance of documented bacterial infections in patients with cirrhosis and secondly to assess the prognostic 
significance of infection in relation to current prognostic scores and ascertain if infection could be considered per se a distinct clinical stage in the natural history of cirrhosis in a welldefined cohort of consecutive hospitalized patients. 


\section{METHODS:}

We evaluated retrospectively all patients with cirrhosis consecutively admitted to the Hepatology ward of a tertiary referral liver center over a 2-year period, from $1^{\text {st }}$ January 2007 to $31^{\text {st }}$ December 2008. Inclusion criteria were diagnosis of cirrhosis based on history, clinical, histological, biochemical and imaging features. We excluded patients with a hospital stay of less than 48 hours, patients with human immunodeficiency virus (HIV) infection or concomitant myeloproliferative disease, patients with HCC outside the Milan criteria and those with previous liver transplantation (OLT). We followed-up patients until OLT or death and the latest follow-up data were collected in 31/10/2009 using the national health systemintegrated hospital register.

The following variables were collected from hospital and laboratory based information systems: sex, age and etiology of cirrhosis; severity of liver disease, components of Model of End-stage Liver Disease (MELD) and United Kingdom Model for End-stage Liver Disease (UKELD). We evaluated blood test results on admission and during hospitalization. Bacterial cultures of blood, urine, peritoneal and pleural fluid were performed whenever infection was suspected based on clinical and/or laboratory signs, and all positive cultures were recorded. Patients with ascites or pleural effusion had a diagnostic tap performed for cell count and cultures when appropriate. According to the aetiology of cirrhosis, the patients were classified into three groups: viral hepatitis (HBV and HCV), alcohol and all other aetiologies.

According to international criteria, diagnosis of infection was based on the following: Spontaneous bacterial peritonitis or spontaneous empyema (SBP and SE) were defined as a neutrophil cell count in ascitic and pleural fluid $\geq 250 / \mathrm{mm}^{3}$ respectively, or positive culture (8). A clean-catch midstream urine specimen for culture and chemical analysis was collected, when urinary tract infection (UTI) was suspected, and UTI was diagnosed as follow: a) urine leukocyte count $\geq 10$ cells $/ \mathrm{mm}^{3}$ and positive urine culture, b) fever, pyuria $(\geq 10$ 
leukocytes $/ \mathrm{mm}^{3}$ ) and $\geq 100,000 \mathrm{CFU} / \mathrm{ml}$, or c) uncountable leukocytes without positive culture (9). In patients with documented or suspected pneumonia, bronchial secretions were collected as appropriate; in case of negative cultures, pneumonia was diagnosed if there was radiological evidence of pulmonary infiltration combined with at least two of the following criteria: fever $>38^{\circ} \mathrm{C}$ or hypothermia $<35.5^{\circ} \mathrm{C}$, dyspnea, cough and purulent sputum, pleural chest pain, or signs of consolidation on physical examination. Other body fluids, like bile, and swabs were cultured when an infection was clinically suspected and infections were diagnosed according to clinical, radiological, and microbiologic data following standard criteria (10). Screening swabs for MRSA were not evaluated unless a clinically relevant (bloodstream or wound) MRSA infection was diagnosed. Infections were considered community acquired (CA) when diagnosed at admission or developed within 48 hours after hospitalization and nosocomial when the diagnosis was made thereafter (11). Health-care associated (HCA) infections were not defined, as this study was planned before this classification was routinely used in Hepatology practice $(12,13)$.

\section{STATISTICAL ANALYSIS:}

Categorical variables are presented as number and percentage, while quantitative variables are summarized by mean and standard deviation (SD) if the distribution is normal or median and interquantile range if not.

The end point was death. The follow-up periods began with first admission and ended with death or liver transplantation, censoring the remaining patients at the last follow-up contact at the hospital or with their general practitioner. The latter were contacted for such information. In the first instance, Cox regression analysis was performed and patients who were transplanted were censored as alive at the time of transplantation. We also used competing risk Cox regression analysis, with death and liver transplantation considered as 
competing risks. In order to minimize the probability of selection bias, we repeated the analysis in a cohort of infected and non-infected propensity score matched patients.

Mortality rate/100 person/year was calculated and survival was described with the KaplanMeier method. Uni- and multivariate Cox regression and competing risk Cox regression models were fitted to identify the most important predictors of outcome. Variables with $\mathrm{p}<0.1$ at univariate analysis were checked for collinearity and entered in the multivariate model, using a stepwise forward approach. Each patient was considered once in the analysis irrespective of the number of hospital admissions. Among patients with more than one hospital admissions, the date of first admission was analyzed for patients without infection, whereas for patients with infection we considered the admission with the first diagnosis of infection. In order to address immortal time bias, additional analyses were performed by classifying the infection event as a categorical time-dependent covariate in Cox survival models (14).

We carried out a propensity analysis using logistic regression to create a score for the infected and non-infected patients. This model included MELD, hepatocellular carcinoma, patients transplanted, reasons of admission (acute decompensation or infection versus others) as well as the interaction term with $\mathrm{p} \leq 0.10$ (MELD $\times$ reasons of admission). We used nearestneighbour matching method with no replacement to match patients in the non-infected group with those in the infected group, with a calliper width of 0.2 of the standard deviation of the logit of the propensity score. After matching appropriate non-parametric paired tests were used (Wilcoxon signed-rank test for continuous variables and McNemar test for dichotomous variables).

All analyses were performed using SPSS version 22.0 for Windows (IBM, New York, NY, USA), except from the competing risk analyses, which were performed using Stata version 12.1 (Statacorp, College Station, Texas, USA). 


\section{RESULTS:}

\section{Patients}

Over the study period, 501 patients with cirrhosis were consecutively admitted to our hospital for more than 48 hours. Of these, 84 patients underwent OLT (18 in the infected group) and were censored as alive at the time of the transplant for the traditional survival analysis, while there was no follow-up information beyond admission for 14 (8 in the noninfected and 6 in the infected group). Patients on the liver-transplant list and patients with HCC were equally distributed among infected and non-infected patients. Further admissions occurred in 280 patients during the study period (153 patients had 2, 127 patients had 3 or more admissions) for a total of 781 different hospital admissions. Mean follow-up was 12.7 (SD 10.4) months.

There were 317 males and 184 females, with a mean age of 53.3 (SD 12.3). Most patients had severe liver disease $(85 \%$ MELD>10 and $56 \%>15$, while $80.5 \%$ were Child-Pugh score B or C); mean admission MELD was 17.1 (SD 6.9) and UKELD was 55.6 (SD 6.4). The aetiology of cirrhosis was alcohol in 242 (48.3\%), viral (HBV or HCV-related) in 114 (22.8\%), and non viral-non alcohol in 145 (28.9\%) cases, including 32 patients with cryptogenic cirrhosis and 15 with NASH. Forty-four patients had HCC and their distribution was even in the infected and non-infected groups. Table 1 shows main clinical characteristics and laboratory findings at admission of the total cohort, in infected and non-infected patients. The characteristics of propensity score matched infected and non-infected patients are shown in the Appendix. The mean MELD score of patients with infection at discharge did not significantly differ from their MELD score at admission $(19.7 \pm 9.1$ vs. $18.7 \pm 7.3, P=0.35)$ and was stable in $35 \%$ of the infected patients, improved in $32 \%$ and deteriorated in $33 \%$. 
Patients were hospitalized on account of decompensation of liver disease in $59.1 \%$ of cases, particularly for tense ascites (17.6\% of cases), active gastrointestinal bleeding (18.6\%), hepatic encephalopathy (9.4\%) or jaundice (13.6\%). Presence of overt infection accounted for $10.4 \%$ of the reasons of admission and elective procedures such as TIPS assessment and placement, loco-regional therapies for hepatocellular carcinoma (HCC), pre-transplant workup or clinical reason not related with decompensation of liver disease for the remaining $30.5 \%$ of cases.

\section{Hospital stay and prevalence of infection}

Median length of in-hospital stay was 10 days (IQR 12.0), with a significant difference between infected and non-infected patients (25.1 vs. 10.2 days; $\mathrm{p}<0.001$ ). 49 patients were transferred from the ward to intensive care (ITU) and stayed there for a median of 7 days (IQR 10).

On a total of 781 admissions in 501 different patients, the prevalence of infection was $25.6 \%$ $(n=200)$. In total, $60 \%(n=120)$ of infections were CA (in 54 cases infection was already overt at admission, the remaining developed symptoms within 48 hour) and $40 \%(n=80)$ were nosocomial infections. There were two patients with bacterascites who were not treated with antibiotics and were considered as non-infected patients. In 52 patients, there were no positive cultures and diagnosis was based on a combination of clinical, biochemical and radiological criteria: 9 cases of pneumonia, 14 of SBP, 1 of UTI and 27 cases with other sites of infections (biliary source, soft tissues). Bacterial isolation was more prevalent in nosocomial than CA infections (98.8\% versus 57.5\%; $\mathrm{p}<0.001)$.

Aetiology and predictors of infection 
Characteristics and factors associated with the presence of infection were evaluated on the total of 781 admissions. 148/200 (74.4\%) infection cases were culture-positive bacterial infections; in some cases, multiple sites or multiple bacteria where isolated in the same patients for a total of 259 positive cultures. The majority of positive cultures involved grampositive cocci (58.1\%). The most common sites of bacterial isolation were blood, ascitic fluid and urine with 73 (28.2\%), 71 (27.4\%) and 37 (14.3\%) positive cultures respectively. Prevalence of multi-resistant strains was $23.1 \%$, and there was no difference among community-acquired and hospital-acquired infections (20.2\% (n=24) vs. $27.5 \%(n=22) ; P$ 0.236). Methicillin-resistant Staphylococcus Aureus, MRSA ( $\mathrm{n}=22)$, was the most frequently isolated multi-resistant strain, followed by Extended Spectrum $\beta$-Lactamase, ESBL $(\mathrm{N}=20)$, gram negative multiresistant bacteria such as Pseudomonas aeruginosa, citrobacter freundii, serratia marcescens and stenotrophomonas maltophilia $(n=5)$, acinetobacter baumanni $(n=$ 3) and vancomycin resistant enterococcus $(n=2)$. A summary of the principal characteristics of infections and cultures is presented in Table 2 .

Factors associated with infection are presented in the Appendix.

\section{Outcomes}

Analysis of outcomes was conducted considering only one admission per patient, either the first or the one with a diagnosis of infection.

190 patients died from the date of first admission, of which 52 died in-hospital, in a mean follow-up of 12.7 (SD 10.4) months.

In-hospital mortality rate was higher in those patients who presented with infection and/or developed infection during hospitalization (HR 5.02; 95\%CI: 2.75-9.16; p<0.001). Also, mortality was higher in this group even for patients who were discharged from hospital (HR 1.59; 95\%CI: 1.12-2.25; p 0.009) and it remained so after excluding early deaths $(\mathrm{n}=44)$, i.e. 
deaths that occurred less than 30 days from admission (HR 1.78; 95\%CI: 1.28-2.49; $\mathrm{p}=$ $0.001)$

Variables which were significantly associated with both 30 days (see appendix) and overall mortality (see table 3) according to univariate analyses were diagnosis of infection, reason of admission acute decompensation of liver disease or infection, more severe disease (UKELD, MELD and Child-Pugh score non-A), ITU stay, advanced age and longer hospital admissions.

Mean survival of patients who were diagnosed with infection during hospitalization was 16.8 months, compared to 25.5 months of patients who didn't have an infection (HR 2.14; 95\%CI: 1.61-2.85; $\mathrm{P}<0.001)$. There was no significant difference between mortality in patients with resistant bacteria infections $(n=24 / 34)$ compared with non-resistant infections $(n=63 / 126)$, with a mean survival of 12.2 vs. 18.1 months respectively (HR: 1.53 ; 95\%CI: 0.95-2.44; p 0.077). Furthermore, no difference in survival between patients with CA and HA infections was seen $(p=0.48)$.

Variables significant at univariate analysis were entered into logistic regression models to assess their relationship with the end-point. As before, due to co-linearity UKELD and reason of admission were not included in the model. Of the 118 patients who were admitted for reasons other than acute decompensation or infection, 21 developed an infection. Infection was significantly associated with overall mortality in this subgroup (HR 1.381, 95\%CI 1.178-1.618). Variables independently associated with higher 30-day mortality rate were the presence of infection at admission and/or during hospitalization (OR 2.91; 95\%CI: 1.48-5.70; p 0.002), MELD score (OR 1.11; 95\%CI: 1.07-1.15; p < 0.0001), ITU stay (OR 2.76; 95\%CI: 1.42-5.34; p 0.003) and repeated admissions (OR 2.27; 95\%CI: 0.995.24; p 0.054) (see Appendix).

Variables independently associated with higher overall mortality rate were the diagnosis of bacterial infection (HR 1.79; 95\%CI: 1.33-2.41; p < 0.001), MELD score (HR 1.09; 95\%CI: 1.07- 
1.11; $\mathrm{p}<0.001$ ), ITU stay (HR 1.67; 95\%CI: 1.10-2.53; p 0.017) and age (HR 1.03; 95\%CI: 1.02-1.04; $\mathrm{p}<0.001)$. All the above is summarized in Table 3 . The same predictors of mortality were significant when competing risk analysis was used in the whole cohort (Table 3), in propensity score matched infected and non-infected patients (Table 4) and when immortal time bias was taken into account for patients who developed an infection after their first admission (Appendix).

When multivariate analysis was repeated excluding early deaths i.e. only including patients who survived at least 30 days after the episode of infection, MELD, history of infection and age were independent predictors of mortality (Table 5). Predictors of survival did not change when competing risk analysis was performed in the whole cohort (Table 5), in propensity score matched infected and non-infected patients (Appendix) and when immortal time bias was taken into account for patients who developed an infection after their first admission (Appendix).

Survival rates at 3, 6, 12 and 30 months were $83 \%, 75 \%, 68 \%, 59 \%$ in patients without diagnosis of infection, versus 55\%, 51\%, 45\% and 39\% in patients with diagnosis of infection. Survival rate at 3, 6, 12 and 30 months was 92\%, 87\%, 79\% and 66\% in those with MELD <15 and $67 \%, 61 \%, 57 \%, 45 \%$ in those with MELD $\geq 15$.

Survival was worse in patients with bacteremia/SBP/pneumonia (14.2 months) compared to patients with other infections (20.8 months).

Figures show the Kaplan Meier survival curves in patients with and without diagnosis of infection (Fig. 1) and stratified by MELD < or $\geq 15$ and diagnosis of infection (Fig. 2).

In all stages of disease, mean survival was significantly lower in infected versus non-infected patients (log rank test for all analyses). In particular in patients with MELD $<15$, mean survival was 19.0 (15.3-22.8) vs. 27.1 (25.1-29.1) months, in those with and without infection 
respectively. Likewise, in patients with MELD $\geq 15$, mean survival was 14.9 (11.6-18.1) months vs. 20.9 (18.5-23.2) in those with and without infection respectively $(\mathrm{P}<0.001)$. Results were similar across all Child-Pugh classes: In 88 patients with Child-Pugh A, mean survival was 22.3 (15.4-29.2) vs. 27.1 (24.5-29.7) months in those with and without infection respectively $(\mathrm{P}=0.165)$, with no deaths occurring within the first month after the infective episode. In 213 patients with Child-Pugh class B mean survival was 19.6 (15.7-23.5) vs. 25.5 (23.3-27.7) months in patients with and without infection, respectively $(P=0.002)$, while in 158 patients with Child-Pugh class C mean survival in these groups was 12.2 (8.5-15.9) vs.17.2 (13.8-20.6) months $(\mathrm{P}=0.023)$. 


\section{DISCUSSION}

In this study, we have shown for the first time that patients with cirrhosis who become infected have a greater risk of death even if they survive the acute episode of infection, independently of the severity of their underlying liver disease. We have further provided updated data on the prevalence and microbiology of bacterial infections in this setting.

We have included consecutive patients over a 2-year period and have followed them up for a median time of over 1 year. We have employed strict criteria to define bacterial infection in order to obtain robust data on the influence of infection. Our findings are striking in the sense that a single episode of infection was independently associated with survival over and above the scores used in transplant centers and degree of liver disease severity. Indeed, the occurrence of bacterial infection for a similar MELD score confers a very different prognosis, and in all stages of disease survival was significantly lower in infected patients than noninfected. Importantly patients with MELD scores $<15$ and infection, have a mortality rate very similar to those with MELD score $\geq 15$ and no infection. Even when patients who did not survive the acute episode of infection were censored, an anamnestic history of infection still represented an independent predictor of survival.

These results obtained in a strictly defined cohort of patients confirm the previous findings of our meta-analysis of 178 studies showing that infections increase mortality in cirrhosis 4-fold, with $30 \%$ of patients dying within one month after infection and $30 \%$ dying within one year (3). This meta-analysis included cohorts with different definitions of infection and varying disease severity, therefore important questions were left unanswered. Most importantly, we could not ascertain if patients with cirrhosis who get infected have a greater risk of death, or if patients with cirrhosis at risk of death get infections in the process of dying. Data from this study point towards the first answer and suggest that infection alters the natural history of cirrhosis and confers a higher risk of death. This effect is independent of the Child-Pugh or the 
MELD score and was recently described in a cohort of patients with compensated cirrhosis due to viral hepatitis (15). Therefore, infection should be regarded as a separate stage of cirrhosis and possibly be added to the list of decompensating events, similar to ascites and variceal bleeding as was previously suggested $(3,16,17)$.

The cause of a high prevalence of infections in patients with cirrhosis is double: firstly patients with advanced chronic liver disease have severe disturbances in the defense mechanisms against bacterial infections (a depressed function of the reticuloendothelial system, neutrophil dysfunction, reduced serum complement and low bactericidal opsonin activity, monocyte deactivation) (18). Secondly, bacterial translocation from the intestine increases susceptibility to infection and becomes clinically significant when it results in spontaneous bacterial peritonitis (SBP) and/or bacteraemia. Infection may lead to a systemic inflammatory response syndrome and sepsis, further deteriorating the circulatory disturbance in cirrhosis, and increasing the risk to develop renal failure, encephalopathy and death. Infection, even after the resolution of an acute episode, may maintain increased levels of endotoxins, bile acids, nitric oxide, carbon monoxide, and cytokines such as tumor necrosis factor and interleukin-6 $(1,19)$. Indeed, the levels of these pro-inflammatory mediators may not return to baseline after resolution of the actual infection thus promoting a cascade of events that culminates in liver failure. Alternative or additional reasons have been proposed to explain the high mortality consequent to bacterial infections in cirrhosis, such as a cytokine-mediated septic cardiomyopathy (20) and the adrenal dysfunction that accompany the liver disease (21). It is also possible that an anamnestic infective episode further worsens or induces immune-paresis and macrophage and neutrophil dysfunction, thus worsening survival (22).

In this study we confirmed the high prevalence of infections $(26.2 \%)$ in hospitalized patients with cirrhosis as reported by other studies $(2,12,23-25)$, and also that bacterial infections 
worsen the prognosis of hospitalized patients with cirrhosis thus increasing mortality. We have also provided data beyond 30-day mortality that has been usually provided in such studies $(23,26)$. It should be noted that the prevalence of infections has not decreased over the past 15 years, despite a more advanced knowledge of the responsible mechanisms and the possibility for an early diagnosis. On the contrary, infections caused by multidrug resistant bacteria (MDRB) are increasing, and can lead to worse outcome (2). Indeed, the prevalence of this type of infection was over $20 \%$ in our cohort; although we did not show a statistically difference in mortality between MDRB and non-MDRB infections, this is probably due to a type I error as the mean survival difference was 5 months in these groups.

These findings have important clinical implications. For patients on the transplant-waiting list, organ allocation systems using minimal listing criteria may disadvantage patients with recent bacterial infections but lower MELD or UKELD scores. Indeed, although such scores allow for the allocation of organs to patients in greatest need, as they are reliable measures of mortality risk in patients with end-stage liver disease, they do not take into account a history of complications including infection and may not accurately reflect the adverse impact of bacterial infection on survival in patients with cirrhosis.

In accord with the hypothesis that occurrence of bacterial infection represents a further prognostic stage, our study confirms that mortality in a large population of patients with cirrhosis is strongly increased by any type of bacterial infection, regardless of the severity of liver disease. The development of infection therefore is independent of the clinical classification of cirrhosis and likely depends on individual factors, such as genetic predisposition, intestinal permeability, bacterial translocation and immune activation. Along these lines, the development of biomarkers of susceptibility to infection will help to identify patients at risk and intervene at an earlier stage. Indeed, an earlier recognition and treatment of infection may be of great value to reduce mortality in these patients. Furthermore, new 
studies of primary and secondary prevention of infections beyond SBP are needed to improve outcomes, similar to the seminal ones for SBP (18).

The main limitation of this study is its retrospective design, although we did include consecutive patients and we were able to obtain all required data from the patients' notes based on a predetermined protocol. Furthermore, we performed a sub-analysis on propensity score matched infected and non-infected patients. Our study cohort cosists of patients hospitalized in 2007-2008, therefore is not contemporary. However, there have been no major changes in the standard of care of decompensated patients since then and the magnitude of the effect of infections in survival is such that the results are pertinent for contemporary cohorts. We could not analyze separately patients that fulfilled the criteria for acute-on-chronic liver failure, as this term had not been introduced when this study was designed and data were captured (27). Finally, we didn't consider the category of HCA infections, which was only recently introduced in hepatology practice (12).

In conclusion, we demonstrated that infection most likely represents a distinct prognostic stage of cirrhosis, which affects survival irrespective of disease severity. Our findings suggest that the occurrence of infection, even with recovery, should be regarded as a prognostic stage of cirrhosis beyond the decompensated stages and the term critically ill cirrhotic could be used for such patients. Future prospective studies should be directed at verifying the importance of this stage and evaluating parameters allowing the identification of infected patients with an increased risk of worse outcome in the short-term. From a more therapeutic point of view, studies addressing the primary and secondary prevention of infections other than SBP are needed to improve outcomes or to facilitate a smoother management towards liver transplantation. 


\section{References}

1. Jalan R, Fernandez J, Wiest R, et al. Bacterial infections in cirrhosis: a position statement based on the EASL Special Conference 2013. J Hepatol 2014;60:1310-24.

2. Fernandez J, Acevedo J, Castro M, et al. Prevalence and risk factors of infections by multiresistant bacteria in cirrhosis: a prospective study. Hepatology 2012;55:1551-61.

3. Arvaniti V, D'Amico G, Fede G, et al. Infections in patients with cirrhosis increase mortality four-fold and should be used in determining prognosis. Gastroenterology 2010;139:1246-56, 1256 e1-5.

4. Kamath PS, Wiesner RH, Malinchoc M, et al. A model to predict survival in patients with end-stage liver disease. Hepatology 2001;33:464-70.

5. Cholongitas E, Marelli L, Kerry A, et al. Female liver transplant recipients with the same GFR as male recipients have lower MELD scores--a systematic bias. Am J Transplant $2007 ; 7: 685-92$.

6. Tripodi A, Chantarangkul V, Primignani M, et al. The international normalized ratio calibrated for cirrhosis (INR(liver)) normalizes prothrombin time results for model for endstage liver disease calculation. Hepatology 2007;46:520-7.

7. D'Amico G, Garcia-Tsao G, Pagliaro L. Natural history and prognostic indicators of survival in cirrhosis: a systematic review of 118 studies. J Hepatol 2006;44:217-31.

8. Rimola A, Garcia-Tsao G, Navasa M, et al. Diagnosis, treatment and prophylaxis of spontaneous bacterial peritonitis: a consensus document. International Ascites Club. J Hepatol $2000 ; 32: 142-53$.

9. Sobel JD, Kaye D. Urinary tract infections. In: Mandell GL, Bennet JE, Dolin R, editors. Principles and practice of infectious diseases. 4th ed. New York: Churchill Livingstone; 1995. p. $662-690$. 
10. Fasolato S, Angeli P, Dallagnese L, et al. Renal failure and bacterial infections in patients with cirrhosis: epidemiology and clinical features. Hepatology 2007;45:223-9.

11. Garner JS, Jarvis WR, Emori TG, et al. CDC definitions for nosocomial infections, 1988. Am J Infect Control 1988;16:128-40.

12. Merli M, Lucidi C, Giannelli V, et al. Cirrhotic patients are at risk for health careassociated bacterial infections. Clin Gastroenterol Hepatol 2010;8:979-85.

13. Horan TC, Andrus M, Dudeck MA. CDC/NHSN surveillance definition of health careassociated infection and criteria for specific types of infections in the acute care setting. Am J Infect Control 2008;36:309-32.

14. Giobbie-Hurder A, Gelber RD, Regan MM. Challenges of guarantee-time bias. J Clin Oncol 2013;31:2963-9.

15. Nahon P, Lescat M, Layese R, et al. Bacterial infection in compensated viral cirrhosis impairs 5-year survival (ANRS C012 CirVir prospective cohort). Gut 2015.

16. Fede G, D'Amico G, Arvaniti V, et al. Renal failure and cirrhosis: a systematic review of mortality and prognosis. J Hepatol 2012;56:810-8.

17. Tsochatzis EA, Bosch J, Burroughs AK. New therapeutic paradigm for patients with cirrhosis. Hepatology 2012;56:1983-92.

18. Tsochatzis EA, Bosch J, Burroughs AK. Liver cirrhosis. Lancet 2014;383:1749-61.

19. Navasa M, Follo A, Filella X, et al. Tumor necrosis factor and interleukin-6 in spontaneous bacterial peritonitis in cirrhosis: relationship with the development of renal impairment and mortality. Hepatology 1998;27:1227-32.

20. Alqahtani SA, Fouad TR, Lee SS. Cirrhotic cardiomyopathy. Semin Liver Dis 2008;28:59-69.

21. Fede G, Spadaro L, Tomaselli T, et al. Adrenocortical dysfunction in liver disease: a systematic review. Hepatology 2012;55:1282-91. 
22. Tritto G, Bechlis Z, Stadlbauer V, et al. Evidence of neutrophil functional defect despite inflammation in stable cirrhosis. J Hepatol 2011;55:574-581.

23. Bartoletti M, Giannella M, Caraceni P, et al. Epidemiology and outcomes of bloodstream infection in patients with cirrhosis. J Hepatol 2014;61:51-8.

24. Borzio M, Salerno F, Piantoni L, et al. Bacterial infection in patients with advanced cirrhosis: a multicentre prospective study. Dig Liver Dis 2001;33:41-48.

25. Bajaj JS, O'Leary JG, Reddy KR, et al. Second infections independently increase mortality in hospitalized patients with cirrhosis: the North American consortium for the study of end-stage liver disease (NACSELD) experience. Hepatology 2012;56:2328-35.

26. Cazzaniga M, Dionigi E, Gobbo G, et al. The systemic inflammatory response syndrome in cirrhotic patients: relationship with their in-hospital outcome. J Hepatol 2009;51:475-82.

27. Moreau R, Jalan R, Gines P, et al. Acute-on-Chronic Liver Failure Is a Distinct Syndrome That Develops in Patients With Acute Decompensation of Cirrhosis. Gastroenterology 2013;144:1426-1437. 
Table 1. Demographic and clinical characteristics of included patients $(n=501)$.

\begin{tabular}{|c|c|c|c|c|}
\hline Variable & $\begin{array}{l}\text { All patients } \\
\qquad(n=501)\end{array}$ & $\begin{array}{l}\text { Non-infected } \\
\qquad(n=335)\end{array}$ & $\begin{array}{l}\text { Infected } \\
(n=166)\end{array}$ & $\mathrm{P}$ \\
\hline Age, years (SD) & $53.3 \pm 12.3$ & $53.1 \pm 12.2$ & $53.8 \pm 12.7$ & 0.52 \\
\hline Male gender, n (\%) & $317(63.3)$ & $217(64.8)$ & $100(60.2)$ & 0.32 \\
\hline Etiology & & & & 0.19 \\
\hline Alcohol, n (\%) & $242(48.3)$ & $155(46.3)$ & $87(52.4)$ & \\
\hline Viral (HBV and/or HCV), n (\%) & $114(22.8)$ & $84(25.1)$ & $30(18.1)$ & \\
\hline Other, n (\%) & 145 (28.9) & $96(28.7)$ & $49(29.5)$ & \\
\hline \multicolumn{5}{|l|}{ Clinical scores } \\
\hline Child-Pugh class, n (\%) & +1 & & & $<0.001$ \\
\hline $\mathrm{A}$ & $92(18.4)$ & $75(23.8)$ & $17(10.9)$ & \\
\hline B & $219(46.5)$ & $152(48.3)$ & $67(42.9)$ & \\
\hline $\mathrm{C}$ & $160(34.0)$ & $88(27.9)$ & $72(46.2)$ & \\
\hline MELD score & $17.1 \pm 6.9$ & $16.3 \pm 6.7$ & $18.7 \pm 7.0$ & $<0.001$ \\
\hline UKELD score (SD) & $55.6 \pm 6.8$ & $54.6 \pm 6.5$ & $57.8 \pm 7.0$ & $<0.001$ \\
\hline HCC, n (\%) & $44(8.8)$ & $34(10.1)$ & $10(6)$ & 0.135 \\
\hline \multicolumn{5}{|l|}{ Laboratory data } \\
\hline $\mathrm{WBC}, * 10^{3} / \mathrm{ml}(\mathrm{IQR})$ & $6.8(5.1)$ & $6.4(4.8)$ & $7.7(5.9)$ & 0.022 \\
\hline Serum creatinine, mg/dl (IQR) & $0.8(0.4)$ & $0.8(0.4)$ & $0.8(0.5)$ & 0.026 \\
\hline Serum bilirubin, mg/dl (IQR) & $2.6(5.4)$ & $2.4(5.0)$ & $3.5(7.9)$ & 0.147 \\
\hline INR (SD) & $1.5 \pm 0.69$ & $1.6 \pm 0.7$ & $1.7 \pm 0.6$ & 0.132 \\
\hline C-reactive protein, mg/dl (IQR) & $14(32)$ & $9(20)$ & $26.5(44.8)$ & $<0.001$ \\
\hline Albumin, g/dl (SD) & $3.1 \pm 0.7$ & $3.3 \pm 0.7$ & $2.9 \pm 0.6$ & $<0.001$ \\
\hline
\end{tabular}




\begin{tabular}{|c|c|c|c|c|}
\hline Na, mEq/L (SD) & $136 \pm 6.1$ & $137 \pm 5.5$ & $134 \pm 6.9$ & $<0.001$ \\
\hline Haemoglobin, g/dl (SD) & $11.3 \pm 2.4$ & $11.5 \pm 2.4$ & $10.9 \pm 2.1$ & 0.006 \\
\hline Platelets, ${ }^{*} 10^{6} / \mathrm{ml}(\mathrm{IQR})$ & $121(115)$ & $118(121)$ & $126(102)$ & 0.59 \\
\hline
\end{tabular}

${ }^{*}$ Continuous data are expresses as mean \pm SD or median (IQR) according to distribution normal or not normal respectively.

Abbreviations: SD, standard deviation; IQR, interquartile range; HBV, hepatitis B virus; HCV, hepatitis C virus; MELD, model for end stage liver disease; UKELD, United Kingdom end stage liver disease; WBC, white blood cell; INR, international normalized ratio 
Table 2. Characteristics of cases of infection and cultures among all 781 admissions

\begin{tabular}{|l|c|}
\hline Cases of infection, $N(\%)$ & $200(25.6)$ \\
\hline Infections with $\geq 1$ positive culture & $148(74)$ \\
\hline Nosocomial & $80(40)$ \\
\hline Polymicrobial infections & $59(29.5)$ \\
\hline Isolation of MDR bacteria & $46(23.1)$ \\
\hline Gram-negative & $100(41.8)$ \\
\hline Gram-positive & $139(58.2)$ \\
\hline Sites of bacterial isolation & $73(28.2)$ \\
\hline Bacteremia & $71(27.4)$ \\
\hline Ascites & $9(3.5)$ \\
\hline Pleural fluid & $37(14.3)$ \\
\hline Urine & $69(26.6)$ \\
\hline Other sites (skin infection, biliary & \\
\hline source or no identifiable source) & \\
\hline
\end{tabular}

Abbreviations: MDR, multidrug-resistant; UTI, urinary-tract infections; SBP, spontaneous bacterial peritonitis. 
Table 3. Survival analysis: factors predictive of overall mortality

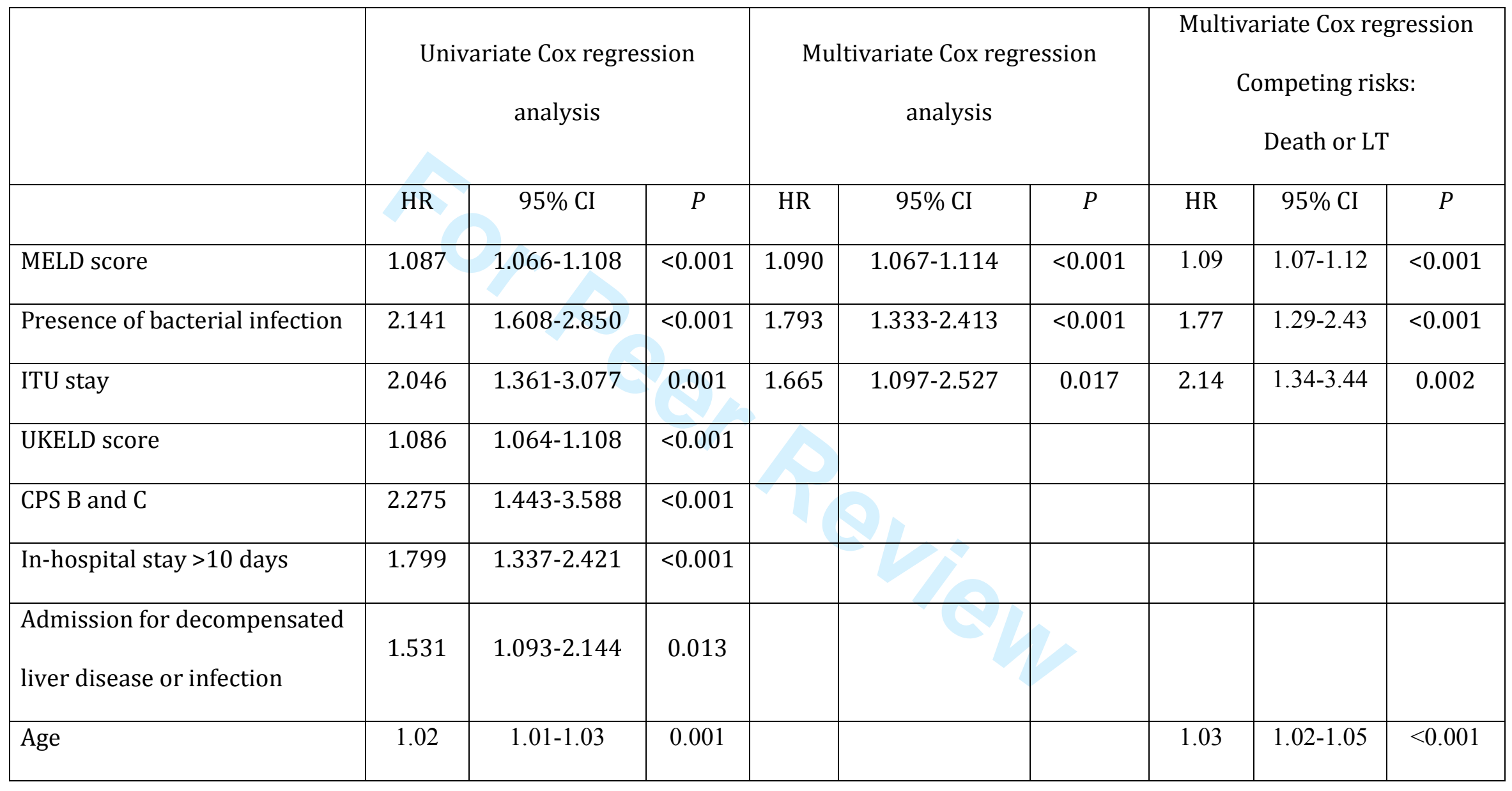


Table 4. Competing risk Cox regression analysis of variables associated with death in total cohort, in propensity score-matched patients (competing risks are death and liver transplantation).

\begin{tabular}{|c|c|c|c|c|c|c|c|c|}
\hline & \multicolumn{3}{|c|}{ Univariate analysis } & \multirow[b]{2}{*}{$P$} & \multicolumn{3}{|c|}{$\begin{array}{c}\text { Multivariate analysis } \\
\text { Competing risks: Death or LT }\end{array}$} & \multirow[b]{2}{*}{$P$} \\
\hline & HR & $\operatorname{se}(\mathrm{HR})$ & $95 \% \mathrm{CI}$ & & HR & se(HR) & $95 \% \mathrm{CI}$ & \\
\hline MELD score & 1.07 & 0.02 & $1.04-1.10$ & $<0.001$ & 1.09 & 0.02 & $1.06-1.13$ & $<0.001$ \\
\hline $\begin{array}{l}\text { History of bacterial infection } \\
\text { (yes) }\end{array}$ & 1.75 & 0.29 & $1.26-2.43$ & 0.001 & 1.65 & 0.31 & $1.15-2.39$ & 0.007 \\
\hline ITU stay (yes) & 2.18 & 0.56 & $1.32-3.60$ & 0.002 & 2.19 & 0.57 & $1.32-3.64$ & 0.003 \\
\hline UKELD score & 1.07 & 0.02 & $1.04-1.10$ & $<0.001$ & - & - & - & - \\
\hline CPS B and C & 1.60 & 0.45 & $0.92-2.79$ & 0.10 & - & - & - & - \\
\hline In-hospital stay $>10$ days & 1.56 & 0.27 & $1.11-2.19$ & 0.01 & - & - & - & - \\
\hline $\begin{array}{l}\text { Admission for decompensated } \\
\text { liver disease or infection (yes) }\end{array}$ & 1.12 & 0.26 & $0.71-1.75$ & 0.63 & - & - & - & - \\
\hline Age & 1.02 & 0.01 & $1.01-1.04$ & $<0.001$ & 1.04 & 0.01 & $1.02-1.05$ & $<0.001$ \\
\hline Admissions $(\geq 2)$ & 1.11 & 0.19 & $0.80-1.54$ & 0.60 & - & - & - & - \\
\hline
\end{tabular}

Abbreviations: ITU, intensive therapy unit; CPS, Child-Pugh score; MELD, model for end stage liver disease; UKELD, United Kingdom end stage liver disease 
Table 5. Survival analysis: factors predictive of mortality excluding early deaths*.

\begin{tabular}{|c|c|c|c|c|c|c|c|c|c|}
\hline & \multicolumn{3}{|c|}{ Univariate Cox regression } & \multicolumn{3}{|c|}{ Multivariate Cox regression } & \multicolumn{3}{|c|}{$\begin{array}{l}\text { Multivariate Cox regression } \\
\text { Competing risks: } \\
\text { Death or LT }\end{array}$} \\
\hline & HR & $95 \%$ CI & $P$ & HR & $95 \% \mathrm{CI}$ & $P$ & HR & $95 \% \mathrm{CI}$ & $P$ \\
\hline MELD score & 1.063 & $1.037-1.089$ & $<0.001$ & 1.068 & $1.040-1.097$ & $<0.001$ & 1.06 & $\begin{array}{l}1.02- \\
1.11\end{array}$ & 0.002 \\
\hline $\begin{array}{l}\text { History of bacterial } \\
\text { infection }\end{array}$ & 1.784 & $1.277-2.492$ & 0.001 & 1.613 & $1.144-2.274$ & 0.006 & 1.67 & $1.06-2.62$ & 0.03 \\
\hline ITU stay & 1.013 & $0.533-1.926$ & 0.969 & & 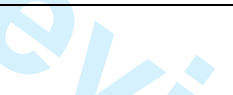 & & & & \\
\hline UKELD score & 1.066 & $1.040-1.092$ & $<0.001$ & & & & & & \\
\hline CPS B and C & 1.775 & $1.115-2.826$ & 0.016 & & & & & & \\
\hline $\begin{array}{l}\text { In-hospital stay }>10 \\
\text { days }\end{array}$ & 1.755 & $1.251-2.463$ & 0.001 & & & & & & \\
\hline $\begin{array}{l}\text { Admission for } \\
\text { decompensated }\end{array}$ & 1.355 & $0.932-1.969$ & 0.112 & & & & & & \\
\hline
\end{tabular}




\begin{tabular}{|c|c|c|c|c|c|c|c|c|c|}
\hline liver disease or & & & & & & & & & \\
\hline Age & 1.022 & $1.009-1.036$ & 0.001 & 1.029 & $1.015-1.044$ & $<0.001$ & 1.03 & $1.02-1.05$ & $<0.001$ \\
\hline$\geq 2$ admissions & 1.491 & $1.071-2.075$ & 0.018 & & & & & & \\
\hline
\end{tabular}

*deaths that occurred in less than 30 days from admission

Abbreviations: ITU, intensive therapy unit; CPS, Child-Pugh score; MELD, model for end stage liver disease; UKELD, United Kingdom end stage liver disease 
Figure 1. Kaplan Meier survival curves in patients with and without diagnosis of infection.

Figure 2. Kaplan Meier survival curves in patients with MELD $<15$ and infection, MELD $\geq 15$ and no infection, MELD $<15$ and infection, MELD $\geq 15$ and no infection 


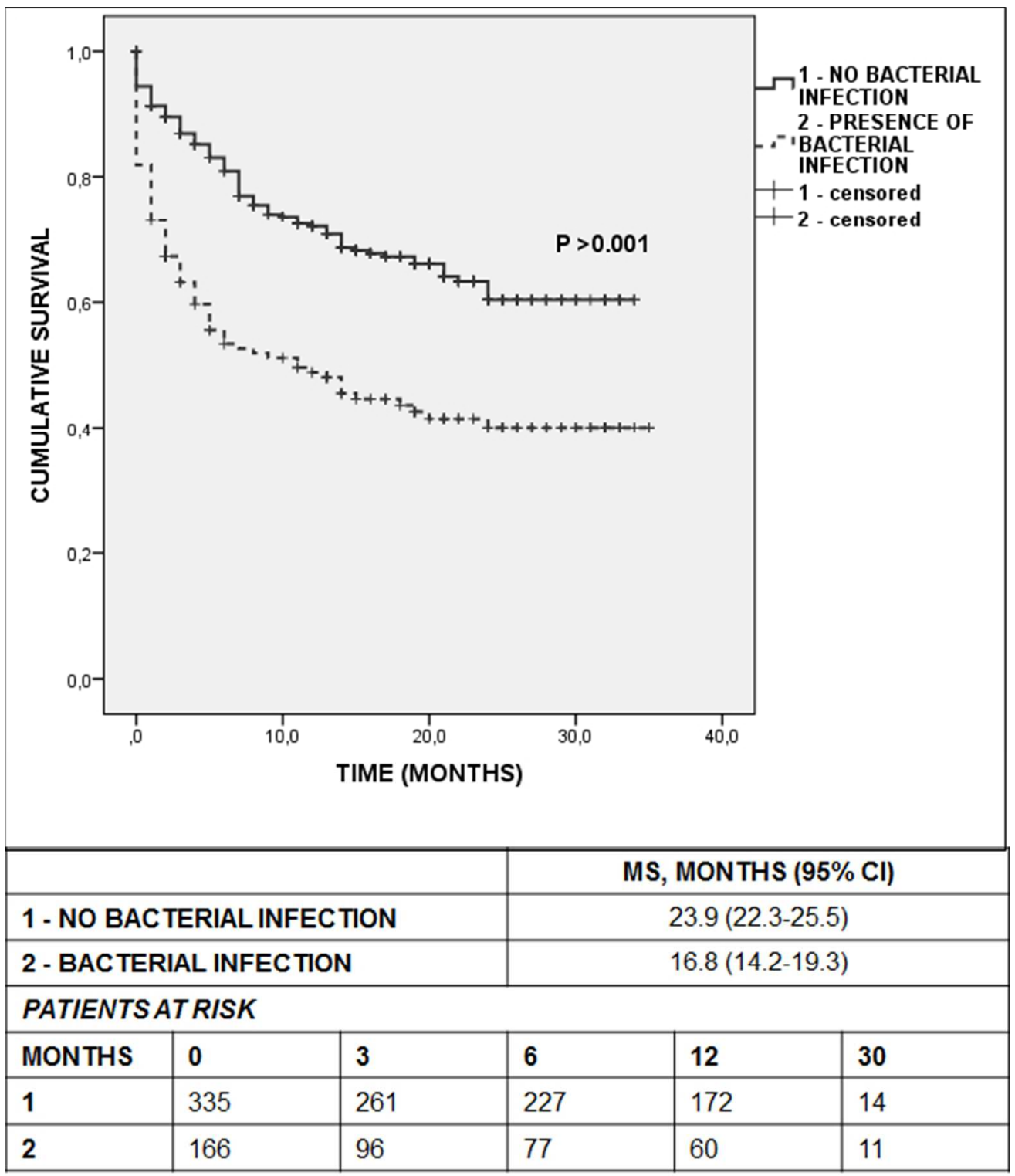

Figure 1

$166 \times 193 \mathrm{~mm}(96 \times 96 \mathrm{DPI})$ 


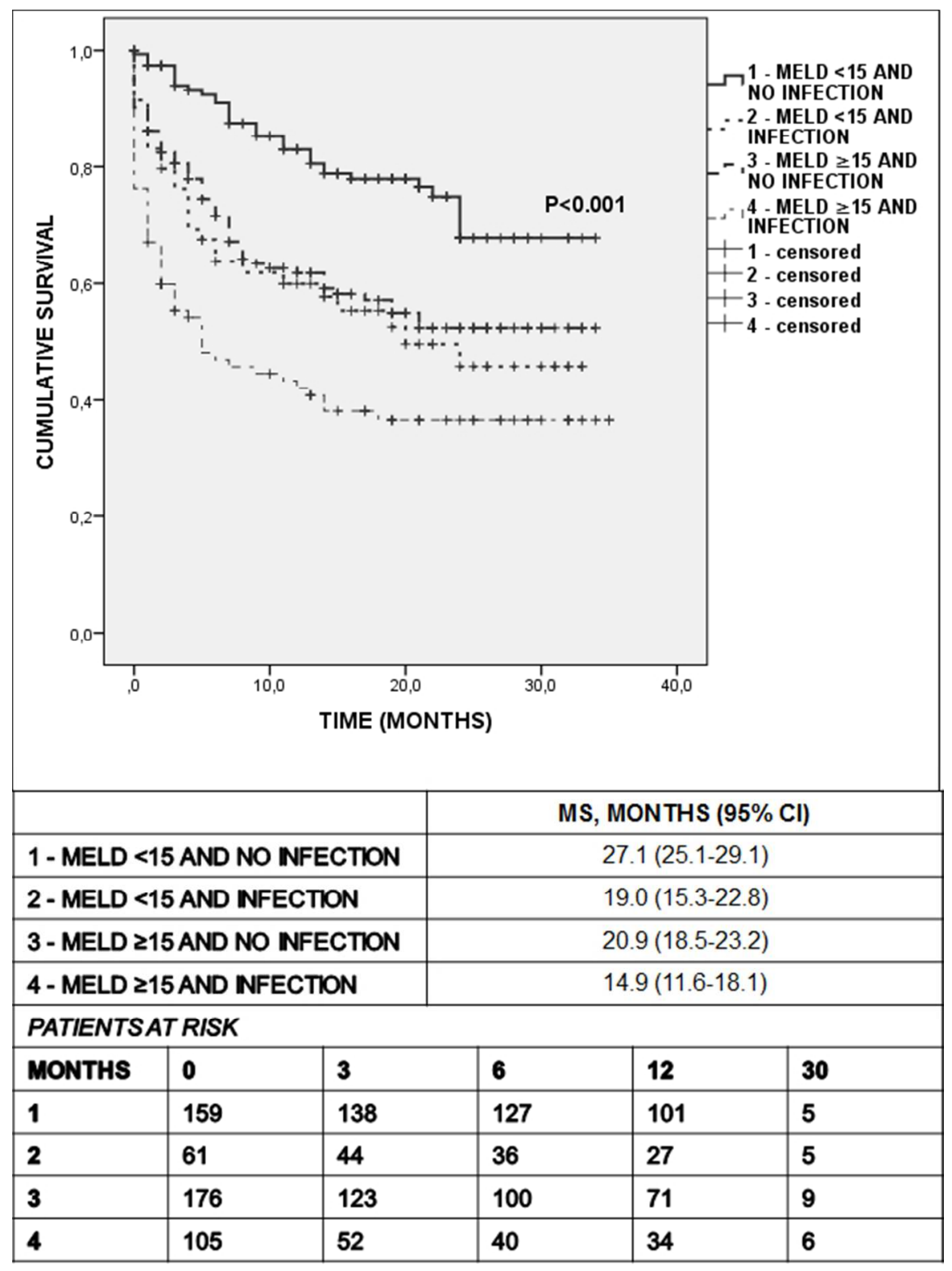

Figure 2

$166 \times 224 \mathrm{~mm}(96 \times 96 \mathrm{DPI})$ 
Web appendix.

Table 1. Propensity score (PS) matching for all patients

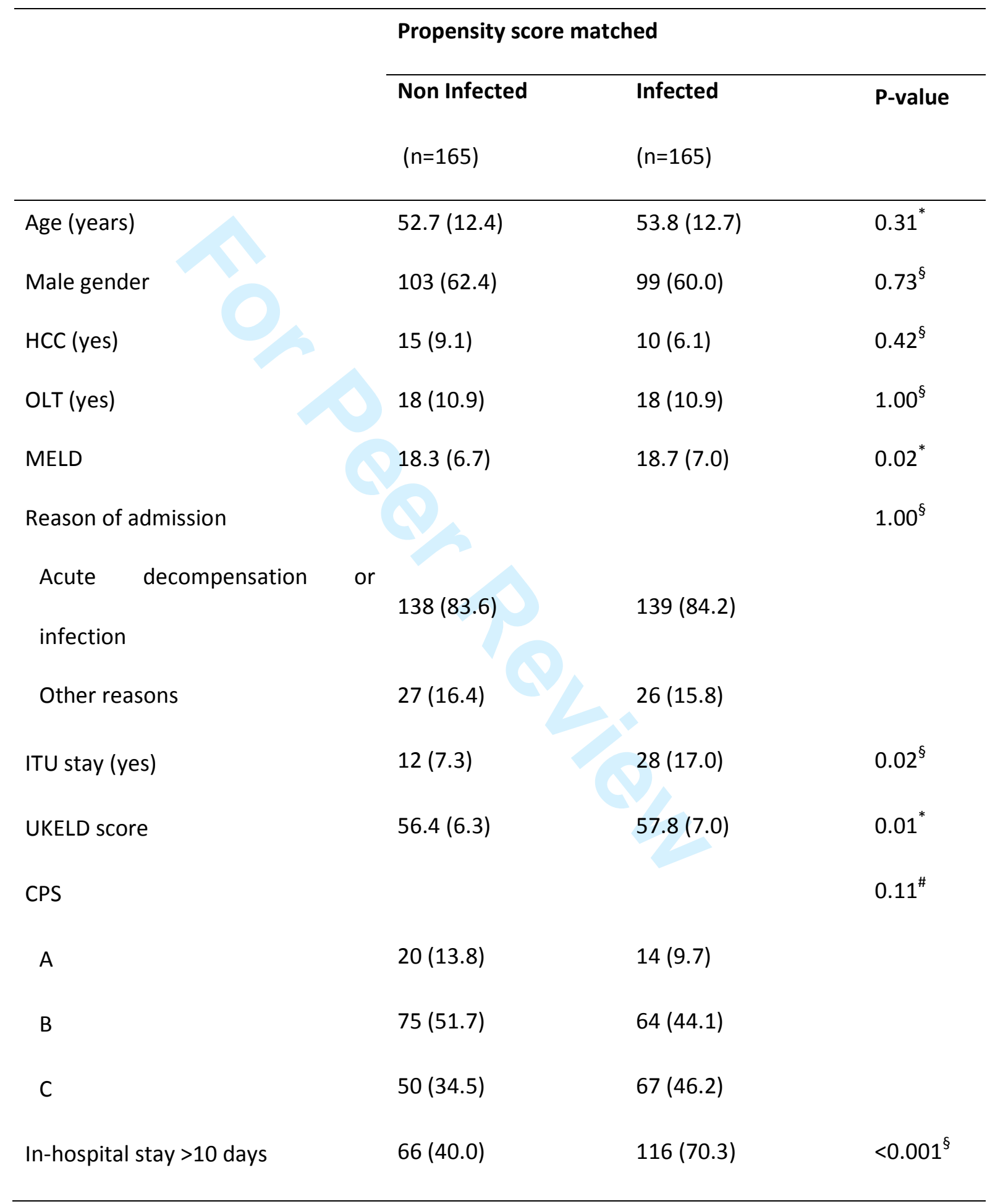


Admissions $(\geq 2)$

$37(22.4)$

$74(44.8)$

$<0.001^{\S}$

Values expressed as mean (SD) and number (per cent) where appropriate.

"Wilcoxon signed-rank test.

${ }^{\S}$ McNemar test.

${ }^{\#}$ McNemar-Bowker test. 
Table 2. Univariate and multivariate analysis of factors associated with infection

\begin{tabular}{|l|c|c|c|c|c|c|}
\hline & \multicolumn{3}{|c|}{ Univariate analysis } & \multicolumn{2}{c|}{ Multivariate analysis } \\
\hline & OR & $95 \% \mathrm{Cl}$ & $P$ & OR & $95 \% \mathrm{Cl}$ & \\
\hline Age & 1.999 & $0.988-1.011$ & 0.922 & & & \\
\hline Sex & 0.879 & $0.644-1.200$ & 0.418 & & & \\
\hline Etiology & 0.953 & $0.797-1.139$ & 0.596 & & & $<0.001$ \\
\hline Number of admissions & 1.829 & $1.347-2.485$ & $<0.001$ & 2.000 & $1.454-2.751$ & \\
\hline$(1$ vs. $\geq 2)$ & & & & & & \\
\hline ITU stay & 1.872 & $1.247-2.810$ & 0.002 & 1.908 & $1.244-2.926$ & NS \\
\hline CPS score non-A & 1.985 & $1.200-3.284$ & 0.008 & - & & 0.020 \\
\hline MELD score & 1.032 & $1.011-1.053$ & 0.003 & 1.026 & $1.004-1.049$ & \\
\hline UKELD score & 1.044 & $1.023-1.066$ & $<0.001$ & & & \\
\hline
\end{tabular}

Abbreviations: ITU, intensive therapy unit; CPS, Child-Pugh score; MELD, model for end stage liver disease; UKELD, United Kingdom end stage liver disease 
Table 3. Survival analysis: factors predictive of short-term mortality at 30 days

\begin{tabular}{|c|c|c|c|c|c|c|}
\hline \multirow[t]{2}{*}{ Factor } & \multicolumn{3}{|c|}{ Univariate Cox regression analysis } & \multicolumn{3}{|c|}{ Multivariate Cox regression analysis } \\
\hline & $\mathrm{HR}$ & $95 \% \mathrm{Cl}$ & $P$ & $\mathrm{HR}$ & $95 \% \mathrm{Cl}$ & $P$ \\
\hline MELD score & 1.130 & $1.094-1.167$ & 0.075 & 1.110 & $1.071-1.150$ & $<0.001$ \\
\hline Presence of bacterial infection & 4.404 & $2.335-8.305$ & $<0.001$ & 2.846 & $1.474-5.498$ & 0.002 \\
\hline ITU stay & 5.456 & $2.952-10.084$ & $<0.001$ & 2.579 & $1.343-4.954$ & 0.004 \\
\hline UKELD score & 1.138 & $1.095-1.183$ & $<0.001$ & & & \\
\hline CPS B and C & 27.885 & $0.973-799.21$ & 0.052 & & & \\
\hline In-hospital stay $>10$ days & 1.925 & $1.032-3.590$ & 0.040 & & & \\
\hline $\begin{array}{l}\text { Admission for decompensated } \\
\text { liver disease or infection }\end{array}$ & 2.988 & $1.263-7.068$ & 0.013 & & & \\
\hline Age & 1.004 & $0.980-1.029$ & 0.743 & & & \\
\hline$\geq 2$ admissions & 2.552 & $1.142-5.706$ & 0.022 & 2.272 & $0.985-5.244$ & 0.054 \\
\hline
\end{tabular}


Table 4. Multivariate Cox regression analysis of variables associated with death by classifying the infection event as a categorical time-dependent covariate to account for immortal time bias.

\begin{tabular}{lccc}
\hline & $\mathrm{HR}$ & $95 \% \mathrm{Cl}$ & $P$ \\
\hline MELD score & 1.10 & $1.07-1.12$ & $<0.001$ \\
Age & 1.03 & $1.02-1.05$ & $<0.001$ \\
ITU stay & 1.89 & $1.22-2.92$ & 0.004 \\
$\begin{array}{lccc}\text { History of bacterial infection } \\
\text { (yes) }\end{array}$ & 2.41 & $1.80-3.24$ & $<0.001$ \\
\hline
\end{tabular}


Table 5. Multivariate Competing risk Cox regression analysis of variables associated with death, in propensity score-matched patients who survived an episode of the infection.

\begin{tabular}{lccc}
\hline & $\mathrm{HR}$ & $95 \% \mathrm{Cl}$ & $P$ \\
\hline MELD score & 1.06 & $1.02-1.11$ & 0.002 \\
Age & 1.03 & $1.02-1.05$ & $<0.001$ \\
$\begin{array}{l}\text { History of bacterial infection } \\
\text { (yes) }\end{array}$ & 1.67 & $1.06-2.62$ & 0.03 \\
\hline
\end{tabular}

Abbreviations: MELD, model for end stage liver disease. 
Table 6. Multivariate Cox regression analysis of variables associated with death in patients who survived an episode of infection. The infection event was classified as a categorical time-dependent covariate to account for immortal time bias.

\begin{tabular}{lccc}
\hline & HR & $95 \% \mathrm{Cl}$ & $P$ \\
\hline MELD score & 1.06 & $1.03-1.09$ & $<0.001$ \\
Age & 1.03 & $1.02-1.05$ & $<0.001$ \\
$\begin{array}{l}\text { History of bacterial infection } \\
\text { (yes) }\end{array}$ & 2.07 & $1.47-2.90$ & $<0.001$ \\
\hline
\end{tabular}


